\title{
Dynamically Consistent Lagrangian Coherent Structures
}

\author{
Francois Lekien ${ }^{* \dagger}$ and Naomi Leonard* \\ ${ }^{*}$ Princeton University, Princeton, NJ 08544 \\ ${ }^{\dagger}$ lekien@princeton.edu
}

\begin{abstract}
In this paper, we compute coherent structures based on radar data collected in Monterey Bay during August 2001 and August 2003. The Lagrangian structures are extracted from a finitetime Lyapunov exponent field. In contrast with earlier approaches, the integration time used in computing the Lyapunov exponents is not constant but adapts to the timescales of the different flow regimes present in the radar data. Nowcasts of the radar data are performed using open-boundary modal analysis (OMA) and the projection coefficients of this filtering method are used to identify periods corresponding to different dynamical regimes in the bay. Lyapunov exponents are computed within a single dynamical regime, hence they determine dynamically consistent coherent structures.
\end{abstract}

\section{INTRODUCTION}

For autonomous and time-periodic two-dimensional systems, the entanglement of the stable and unstable manifold of hyperbolic equilibria or hyperbolic periodic orbits plays a central role in understanding and quantifying mixing and stirring $[1,2,3]$. In recent years, this approach has been extended to aperiodic velocity fields. In such flows, trajectories can have a stable and an unstable manifold only if their linearization possesses an exponential dichotomy $[4,5]$, i.e. the coexistence of an exponential growth and an exponential decay about this trajectory. Similarly, one can derive the existence of a stable and an unstable manifold for those trajectories that have both a positive and a negative Lyapunov exponent. These notions are closely related to the behavior of the solutions of the linearized system for $t \rightarrow \pm \infty$.

In this paper, we study a vector field measured by high-frequency radar along the California coastline. Such a velocity field is known only over a finite interval of time (the measurement period) and any notion involving asymptotic limits in time, such as Lyapunov exponents, hyperbolicity or exponential dichotomies are inapplicable. Recently a finite-time version of Lyapunov exponents was introduced [6] under the name Direct Lyapunov Exponent (DLE). The ridges of such a field determine coherent structures. These structures are the mixing guides [7] for finite-time aperiodic vector fields and are equivalent to invariant manifolds for autonomous systems.

Finite-time Lyapunov exponents are typically computed using a constant integration time [7, 8]. In contrast with this approach, we use modal analysis [9] to determine regimes in the data and adapt the integration time of the Lyapunov exponents to the dynamics of the flow. This method produces coherent structures that are discontinuous at the boundaries between regimes but maintain dynamical consistency inside a regime. 


\title{
FLOW REGIMES IN MONTEREY BAY
}

\author{
High-Frequency Radar Data
}

Our analysis makes use of high-frequency (HF) radar technology [10, 11, 12, 13], which measures near surface currents along coastlines. Such an HF radar installation has been operating in Monterey Bay since 1994 [10, 11]. In our study, we use data from this installation, binned every hour on a horizontal uniform grid with $1 \mathrm{~km}$ by $1 \mathrm{~km}$ intervals.

\section{Open-Boundary Modal Analysis}

Modal analysis $[9,14,15]$ is a technique used to interpolate, extrapolate and filter remote sensing data. The method was originally used in electromagnetism and quantum physics where the solution of a perturbed Hamiltonian is projected onto the eigenfunctions of the unperturbed Hamiltonian. Its application in fluid mechanics raised the issue of open-boundaries. Most coastal problems, such as the domain shown on Fig. 1, are not completely bounded by a coastline. The limited range of the onshore radars creates an arbitrary boundary in the ocean, called an open-boundary. The first attempts to apply modal analysis to domains containing open-boundaries modified the modes to allow inflow and outflow through the open-boundary [15]. However, this procedure creates time-dependent modes [15]. Recent developments [9] show that increasing the number of modes allows for a time-independent basis of the space of all available velocities. In this paper, we use such an Open-Boundary Modal Analysis (OMA). The complete algorithm is extensively described in [9] and the most energetic modes for the bay of Monterey are shown on Fig. 1. At each time, the radar data is projected onto the sequence of modes and the extrapolated velocity field is represented as a linear combination of these time-independent modes

$$
\mathbf{v}(\mathbf{x}, t)=\sum_{i=1}^{n} \alpha_{i}(t) \mathbf{v}_{i}(\mathbf{x}),
$$

where each $\mathbf{v}_{i}(\mathbf{x})$ is a mode of Fig. 1. In this paper, we are only concerned with the time evolution of the projection coefficients $\alpha_{i}(t)$.

\section{Partition in Regimes}

Our objective is to partition the time interval into $N$ regimes $\left[\gamma_{i}, \gamma_{i+1}\right]$, each corresponding to a particular dynamical behavior of the flow in the bay. The spatial modes used in Eq. 1 are autonomous, so changes in regime are entirely contained in the behavior of the coefficients $\alpha_{i}$. For ease of notation, we stack these coefficients in a vector $\bar{\alpha}$. Notice that the dimension of $\bar{\alpha}$ is the number of modes used, hence a function of how much filtering is applied to the data, not the dimension of the domain. 


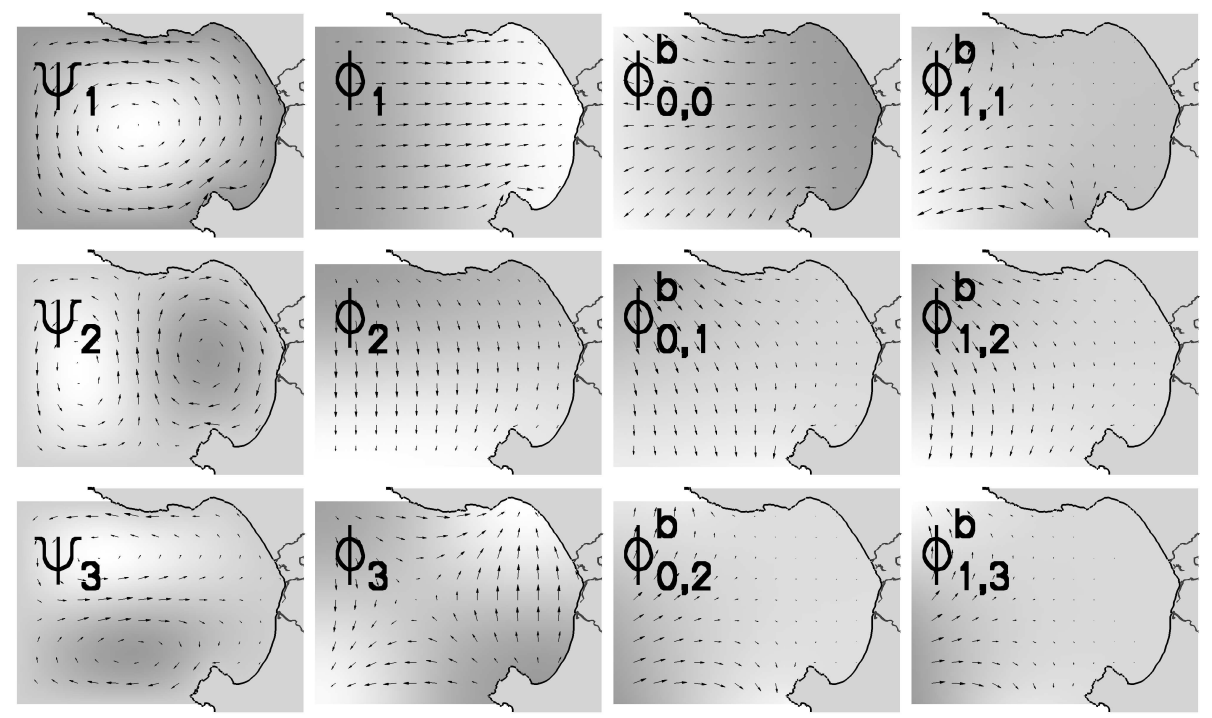

FIGURE 1. Most energetic modes computed for Monterey Bay. See [9] for details.

The partition of the motion of the vector $\bar{\alpha}(t)$ in different regimes can be conducted using many techniques, including Fourier decomposition and direct observation. In this paper, we use a geometric approach and we require that the elected partition minimizes

$$
\xi=\frac{1}{2 N}+N \sum_{i=1}^{N} \int_{\gamma_{i}}^{\gamma_{i+1}} d t \int_{\gamma_{i}}^{\gamma_{i+1}} d \tau\|\bar{\alpha}(t)-\bar{\alpha}(\tau)\| .
$$

For a given $N$, the second term in Eq. 2 reaches a minimum when the partition divides the time interval into subintervals for which the vector $\bar{\alpha}(t)$ stays in a confined region of its phase space. By computing analytically the second term for a uniform distribution $\bar{\alpha}(t)=t$ with $t \in[0,1]$ and a partition $\gamma_{i}=(i-1) / N$, one can see that the second term does not take the same value for all the possible partitions of the uniform distribution. We require that any number $N$ of subintervals can be used in the partitionning of a constant distribution, hence the extra term $1 / 2 N$ in Eq. 2 that makes $\xi$ independent of $N$ for a uniform distribution.

\section{Results}

We performed an OMA nowcast of the radar data collected in Monterey Bay using 20 modes [9] for July, August and September of 2001 and 2003. For each of these years, the minimum of Eq. 2 corresponds to a partition that divides the month of August into 5 qualitatively different regimes. Table 1 gives the endpoints of each of these subintervals. Figure 2 shows the projection of the motion of $\bar{\alpha}(t)$ on several two-dimensional planes. 

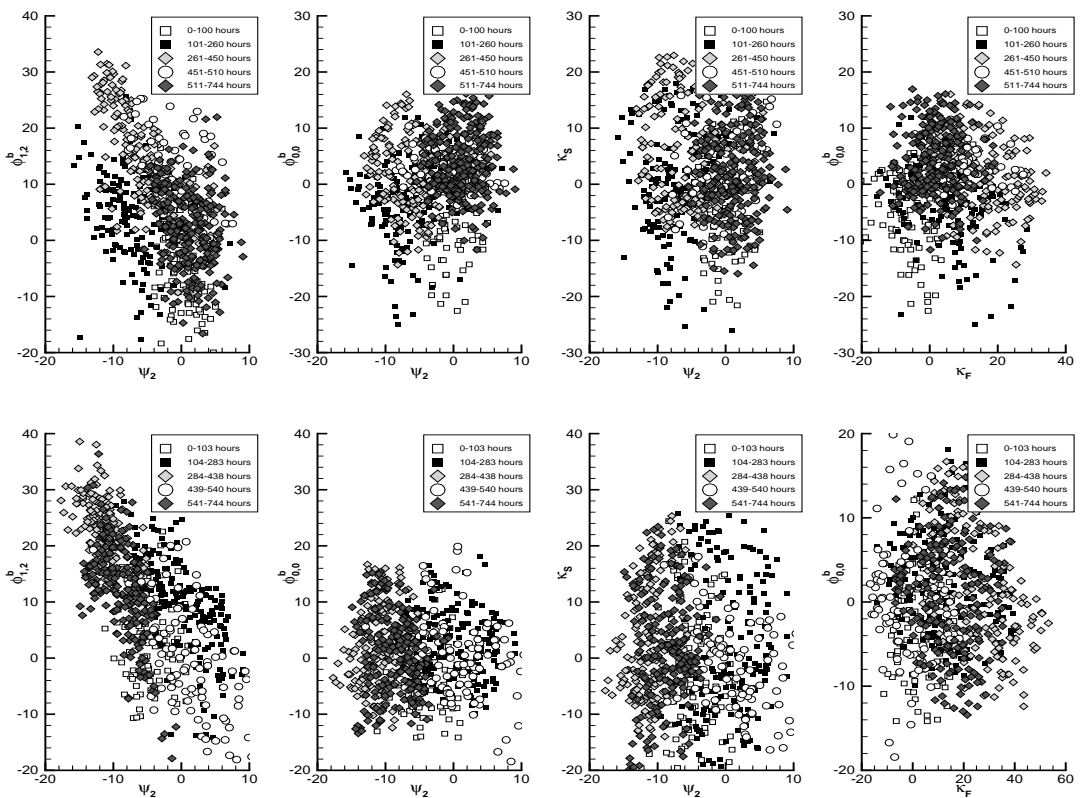

FIGURE 2. Projection of $\bar{\alpha}$ on various two-dimensional planes for August 2001 (top panels) and August 2003 (bottom panels). The style of the points matches the regimes of Table 1. $\kappa_{F}$ is the sum of all modes having inflow on the northern boundary and outflow on the southern boundary. $\kappa_{S}$ is the sum of all modes creating a non-zero divergence. See Fig. 1 for a complete nomenclature of the modes used.

TABLE 1. Partition of August 2001 and August 2003 into dynamical regimes. Times are given in hours since August 1st 0:00 GMT.

\begin{tabular}{|c|c|c|c|}
\hline \multicolumn{4}{|c|}{ August 2001} \\
\hline Date starts & Starts & Stops & Length \\
\hline Aug 1st 00:00 & - & 100 & 100 \\
\hline Aug 5th 05:00 & 101 & 260 & 160 \\
\hline Aug 11th 21:00 & 261 & 450 & 190 \\
\hline Aug 19th 19:00 & 451 & 510 & 60 \\
\hline Aug 22nd 07:00 & 511 & - & 234 \\
\hline
\end{tabular}

\begin{tabular}{cccc}
\multicolumn{4}{c}{ August 2003 } \\
\hline \multicolumn{1}{c}{ Date starts } & Starts & Stops & Length \\
\hline Aug 1st 00:00 & - & 103 & 103 \\
Aug 5th 08:00 & 104 & 283 & 180 \\
Aug 12th 20:00 & 284 & 438 & 155 \\
Aug 19th 07:00 & 439 & 540 & 102 \\
Aug 23rd 13:00 & 541 & - & 204 \\
\hline
\end{tabular}

Negative values of the amplitude of the mode $\psi_{2}$ correspond to a double-gyre pattern in the bay (see Fig. 1). High amplitudes of the modes with divergence indicates an upwelling period. Points corresponding to different regimes are shown with different symbols in Fig. 2. This reveals that the flow in the bay goes through double-gyre regimes (Aug 5th $\rightarrow$ Aug 19th 2001, Aug 12th $\rightarrow$ Aug 19th 2003 and Aug 23rd $\rightarrow$ Aug 30th 2003) followed by periods of relaxation. An in-depth analysis of the regimes can be found in $[10,16]$. 


\section{LAGRANGIAN COHERENT STRUCTURES}

\section{Direct Lyapunov Exponents}

The finite-time Lyapunov exponent map $\sigma_{T}\left(\mathbf{x}_{0}, t_{0}\right)$ is computed using the flow map, i.e, the map that takes an initial fluid particle position $\mathbf{x}_{0}$ at time $t_{0}$ to its later position $\mathbf{x}\left(t_{0}+T ; \mathbf{x}_{0}, t_{0}\right)$ at time $t_{0}+T$. To perform this analysis, a uniform grid of $200 \times 200$ particles is launched at time $t_{0}$. Each particle is advected using a 4th order Runge-KuttaFelhberg algorithm and a 3rd order interpolation [17]. The coastline is modeled as a free-slip boundary and particles that cross the open-boundaries of the domain on the northern, eastern and southern edge are disregarded [18].

These particle trajectories are used to approximate the spatial gradient of the flow map $d \mathbf{x} / d \mathbf{x}_{0}$, from which the Cauchy-Green strain tensor is directly derived according to

$$
S_{T}\left(\mathbf{x}_{0}, t_{0}\right)=\left[\frac{\partial \mathbf{x}\left(t_{0}+T ; \mathbf{x}_{0}, t_{0}\right)}{\partial \mathbf{x}_{0}}\right]^{\top}\left[\frac{\partial \mathbf{x}\left(t_{0}+T ; \mathbf{x}_{0}, t_{0}\right)}{\partial \mathbf{x}_{0}}\right],
$$

where the superscript ${ }^{\top}$ refers to a matrix transpose. The finite-time Lyapunov exponent [19] is then given by

$$
\sigma_{T}\left(\mathbf{x}_{0}, t_{0}\right)=\frac{1}{2 T} \log \left(\lambda_{\max }\left(S_{T}\left(\mathbf{x}_{0}, t_{0}\right)\right)\right) .
$$

\section{Coherent Structures and Flow Regimes}

As shown in $[19,20]$, repelling coherent lines are local maximizing ridges of the scalar field $\sigma_{T}\left(\mathbf{x}_{0}, t_{0}\right)$. The same procedure performed backward in time (i.e., for $T<0$ ) renders attracting coherent structures. The ridges computed are quasi Lagrangian [21]. More specifically, the flux through a ridge of $\sigma_{T}\left(\mathbf{x}_{0}, t_{0}\right)$ is negligible provided that the flow stays in the same dynamical state during the interval of time $\left[t_{0}, t_{0}+T\right]$ for which the flow map is computed.

As a result, there is a severe influence of the parameter $T$ when computing Lyapunov exponents and coherent structures. Notice that large $T$ is not necessarily desirable, as it produces averaged structures and destroys the Lagrangian properties of these structures [21]. Coherent structures are typically computed by estimating or eyeballing an appropriate constant integration time $T[6,8,18]$. In this paper, we propose to use a variable integration time $T\left(t_{0}\right)$. We use the longest possible integration time $T$ for which the interval $\left[t_{0}, t_{0}+T\right]$ remains inside a single dynamical regime. This provides a dynamically consistent finite-time Lyapunov exponent.

Example. To illustrate this procedure and the properties of the dynamic Lyapunov exponent and its associated coherent structures, we consider the one-dimensional linear system $\dot{x}=-\sin t x$.

Lyapunov Exponent. The classical finite-time Lyapunov exponent for this linear system is $\sigma_{T}\left(x_{0}, t_{0}\right)=\frac{1}{T}\left(\cos \left(t_{0}+T\right)-\cos \left(t_{0}\right)\right)$. Figure 3 illustrates how $\sigma_{T}$ depends strongly on the parameter $T$. 


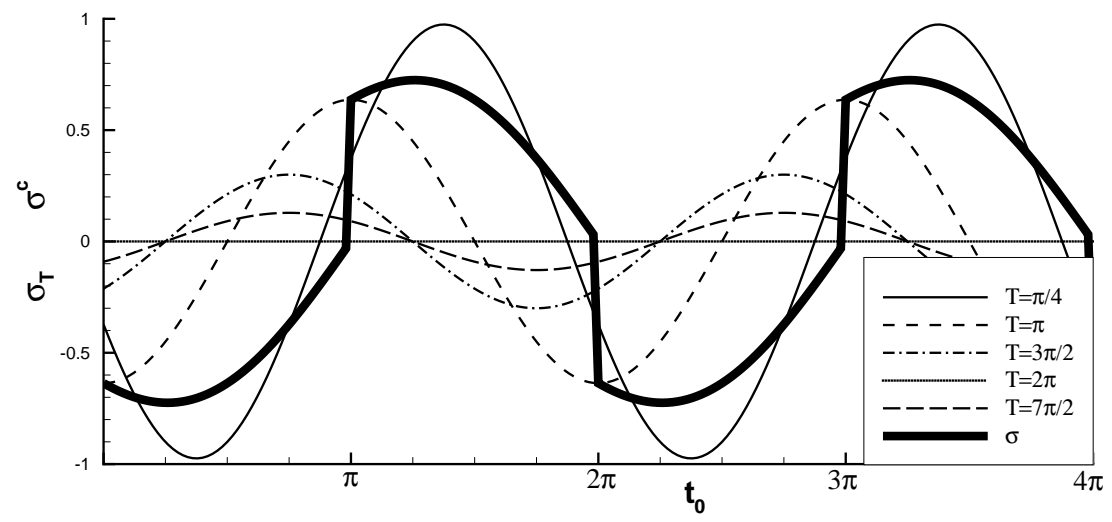

FIGURE 3. Finite-time Lyapunov exponent for $\dot{x}=-\sin t x$. Each light curve corresponds to $\sigma_{T}$ with a different value of the integration time $T$. The heaviest curve corresponds to the dynamically consistent exponent $\sigma^{c}$.

Dynamical Regimes. The system is already decomposed in spatial and temporal modes. The projection coefficient $\bar{\alpha}(t)=-\sin t$ oscillates between -1 and +1 with a probability of presence $P(\bar{\alpha}) d \bar{\alpha}=\left(\pi \sqrt{1-\bar{\alpha}^{2}}\right)^{-1} d \bar{\alpha}$. The two asymptotical peaks correspond to dynamically different behaviors (attractive and repulsive) and we set a boundary between these behaviors at $\bar{\alpha}=0$, i.e. $\gamma_{i}=i \pi$. This corresponds to a partition of the time interval into subintervals of length $\pi$.

Dynamically Consistent Lyapunov Exponent. Based on the partition above, the dynamically consistent finite-time Lyapunov exponent $\sigma^{c}$ corresponds to $\sigma_{\tilde{T}}$, where $\tilde{T}=\pi\left\lceil t_{0} / \pi\right\rceil{ }^{1}$. Hence, $\sigma^{c}\left(x_{0}, t_{0}\right)=\left(\pi\left\lceil t_{0} / \pi\right\rceil-t_{0}\right)^{-1}\left( \pm 1-\cos t_{0}\right)$. Notice that the regime-based exponent $\sigma^{c}$ provides much more insight about the underlying dynamics. Its sign directly determines the stability of the trajectory for a given regime. The cost for dynamical consistency is the discontinuity of the exponent and associated structures at the boundary between regimes.

\section{Results}

Panels (a) and (b) in Fig. 4 shows the coherent structures computed for August 5th, 2001 at 15:00 GMT. Panel (a) corresponds to the dynamically consistent structure and reveals the barrier closing the bay during the double-gyre regime [18]. Its presence results from the strong amplitude of the mode $\psi_{2}$ during this period. Panel (b) shows

${ }^{1}\lceil x\rceil$ denotes the smallest integer that is larger than or equal to $x$. 

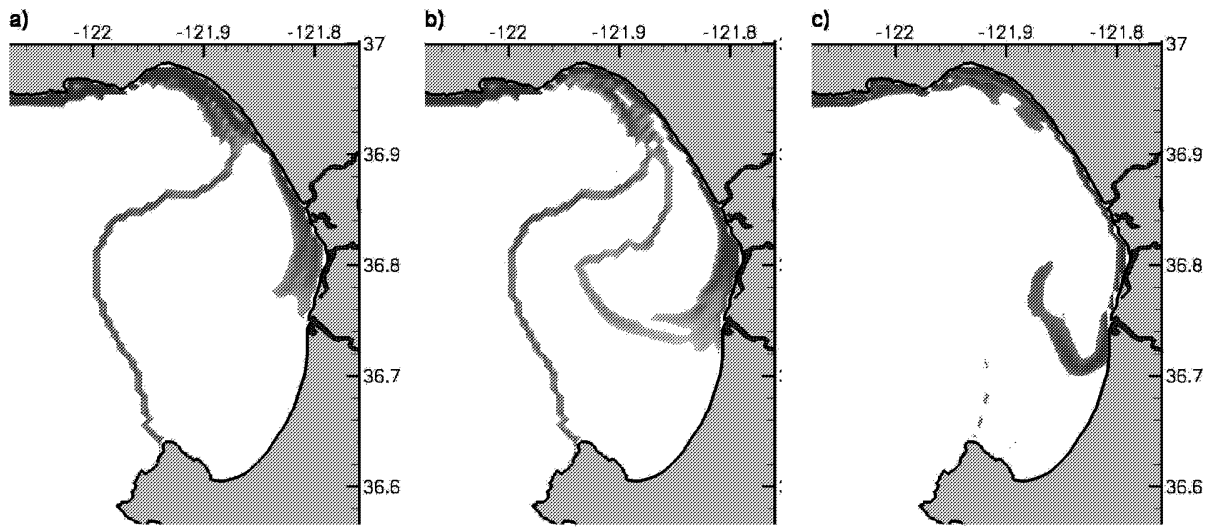

FIGURE 4. a) Dynamically consistent coherent structure for August 5th, 2001 at 15:00 GMT. The integration time has been adapted to match the end of the regime in Table 1. b) Coherent structure for August 5th, 2001 at 15:00 GMT without adaptation of the integration time. c) Dynamically consistent coherent structure for August 13th, 2001 at 12:00 GMT.

the coherent structure obtained when the integration time for the Lyapunov exponent is larger than the remaining of the interval corresponding to this regime. The structure suffers an apparent bifurcation caused by the interference of the coherent structure of the next regime. The characteristic coherent structure for the next regime is shown on panel (c), corresponding to the dynamically consistent exponent for August 13th, 2001 at 12:00 GMT.

\section{CONCLUSION}

In this paper, we used open-boundary modal analysis to divide the high-frequency radar data collected in Monterey Bay, California into dynamical regimes. The definition of finite-time Lyapunov exponent has been modified to allow for variable integration times. A dynamically consistent Lyapunov exponent was computed by selecting the largest integration time $T$, for which the trajectories used to approximate the flow map remain inside the same dynamical regime. The dynamically consistent Lyapunov exponent gives a much clearer representation of the Lagrangian dynamics inside a single flow regime. In contrast with classical finite-time Lyapunov exponents, a discontinuity of the dynamically consistent exponent and its associated coherent structure appears at the boundary between regimes. 


\section{ACKNOWLEDGMENTS}

The authors would like to thank Dr. Jeffrey Paduan and Dr. Michael Cook for their help in using the high-frequency radar data available for Monterey Bay and their useful input in determining and understanding flow patterns in the bay. The normal modes used in this paper were computed using a modified version of Dr. Randolf Bank's PLTMG solver [22].

This research was funded by ONR grant N0014-02-1-0826.

\section{REFERENCES}

1. Rom-Kedar, V., Physica D, 43, 229-268 (1990).

2. Rom-Kedar, V., and Wiggins, S., Physica D, 51, 248-266 (1991).

3. Wiggins, S., Chaotic Transport in Dynamical Systems, Springer-Verlag, New York, 1992.

4. Rogerson, A., Miller, P. D., Pratt, L. J., and Jones, C. K. R. T. J., Journal of Physical Oceanography, 29(10), 2635-2655 (1999).

5. Coppel, W. A., "Dichotomy in Stability Theory," in Lecture Notes in Mathematics, Springer, New York, 1978, vol. 629.

6. Haller, G., Phys. Fluids A, 13, 3368-3385 (2001).

7. Voth, G., Haller, G., and Gollub, J., Phys. Rev. Letters, 88 (25), 254501.1 - 254501.4 (2002).

8. Lekien, F., Coulliette, C., and Marsden, J., "Lagrangian Structures in High-Frequency Radar Data and Optimal Pollution Timing," in Experimental Chaos, American Institute of Physics, 2003, vol. 676, pp. 162-168.

9. Lekien, F., Coulliette, C., Bank, R., and Marsden, J., Journal of Geophysical Research-Oceans (in press, 2004).

10. Paduan, J. D., and Rosenfeld, L. K., Journal of Geophysical Research-Oceans, 101, 20669-20686 (1996).

11. Paduan, J. D., and Cook, M. S., Oceanography, 10, 49-52 (1997).

12. Prandle, D., and Ryder, D. K., Nature, 315, 128-131 (1985).

13. Georges, T. M., Harlan, J. A., and Lematta, R. A., Nature, 379, 434-436 (1996).

14. Lipphardt, B. L., Jr., Kirwan, A. D., Jr., Grosch, C. E., Lewis, J. K., and Paduan, J. D., Journal of Geophysical Research-Oceans, 105(C2), 3, 425-450 (2000).

15. Chu, P. C., Ivanov, L. M., Korzhova, T. P., Margolina, T. M., and Melnichenko, V. M., J. of Atmospheric and Oceanic Tech., 20, 478-491 (2003).

16. Rosenfeld, L. K., Schwing, F. B., Garfield, N., and Tracy, D. E., Continental Shelf Research, 14 (9), 931-964 (1994).

17. Lekien, F., and Marsden, J., Int. Journal for Numerical Methods in Engineering (submitted, 2004).

18. Coulliette, C., Lekien, F., Haller, G., and Paduan J.and Marsden, J., Environmental Science and Technology (submitted, 2004).

19. Haller, G., Physica D, 149, 248-277 (2001).

20. Haller, G., Phys. Fluids A, 14, 1851-1861 (2002).

21. Shadden, S., Lekien, F., and Marsden, J., Physica D (in preparation, 2004).

22. Bank, R. E., PLTMG User's Guide, SIAM, 1990. 